\title{
EVALUATION OF FOUR REAL-TIME PCR ASSAYS FOR DETECTION OF INFLUENZA A(H1N1)V VIRUSES
}

\author{
J Ellis (joanna.ellis@hpa.org.uk)1, M Iturriza1 ${ }^{1}, \mathrm{R}$ Allan ${ }^{1}$ *, A Bermingham ${ }^{1}, \mathrm{~K}_{\text {Brown }}{ }^{1}, \mathrm{~J} \mathrm{Gray}^{1}, \mathrm{D} \mathrm{Brown}^{1}$ \\ 1. Virus Reference Department, Centre for Infections, Health Protection Agency, Colindale, London, United Kingdom
}

\begin{abstract}
The sensitivity and specificity of four real-time PCR assays (HPA $\mathrm{A}(\mathrm{H} 1) \mathrm{v}, \mathrm{CDC} A(\mathrm{H} 1) \mathrm{v}, \mathrm{HPA} \mathrm{A}(\mathrm{N} 1) \mathrm{v}$ and NVRL S-OIV assays) were evaluated for detection of influenza $A(H 1 N 1) v$ viruses. Nose and throat swab samples containing influenza $A(H 1 N 1) v$ viruses, seasonal influenza AH3N2, AH1N1, influenza B viruses, or negative for influenza viruses were tested by the four assays. Specificity was also analysed using influenza A viruses of different subtypes and non-related respiratory viruses. The sensitivities and specificities of the four assays were in a similar range and suitable for diagnostic use. The HPA ( $\mathrm{H} 1)$ v and the S-OIV assays were the most sensitive assays for use as a first line test, but the S-OIV assay was less specific, detecting all avian subtypes of influenza A viruses tested. The results of this study demonstrate that the concurrent use of primary diagnostic and confirmatory assays provides rapid and accurate assessment of confirmed cases, and allows appropriate management of patients.
\end{abstract}

\section{Introduction}

The recent emergence of new influenza $A(H 1 N 1)$ virus (henceforth: influenza $A(H 1 N 1) v$ virus, where v stands for variant, according to nomenclature agreed by the World Health Organization Global Influenza Surveillance Network - WHO GISN) in humans [1-2] has led to the requirement for sensitive and specific assays for the differential diagnosis and confirmation of influenza $A(H 1 N 1) v$ virus infections, necessary to guide public health actions. Real-time PCR is widely considered the gold standard for molecular detection of influenza viruses due to its high assay specificity, sensitivity and broad linear dynamic range. In the present study, the performance (including sensitivity and specificity) of four real-time PCR assays designed to detect influenza $A(\mathrm{H} 1 \mathrm{~N} 1) v$ viruses in respiratory specimens has been evaluated. Two assays are based on detection of haemagglutinin $(\mathrm{HA})$, one on the detection of neuraminidase (NA) and one on the matrix $(M)$ gene.

\section{HPA (H1)v assay}

The influenza $A(\mathrm{H} 1) v$ specific assay of the Health Protection Agency (HPA) contains primers and a dual-labelled TaqMan MGB probe (Applied Biosystems) targeting conserved sequences in the $H A$ gene of $A(H 1 N 1) v$ viruses, and the positive control swine $A(H 1 N 1)$ virus $A / A r a g o n / 3218 / 2009$, in a 1-step TaqMan PCR assay [3]. The advantage of using a genetically distinct positive control virus (A/Aragon/3218/2008) is that false positives can be differentiated by sequence from true positives.

\section{CDC (H1)v assay}

The Centers for Disease Control and Prevention (CDC) realtime RT-PCR kit designed for the detection and characterisation of influenza $\mathrm{A}(\mathrm{H} 1 \mathrm{~N} 1)$ v viruses contains a panel of oligonucleotide primers and dual-labelled hydrolysis probes [4]. The CDC (H1)v primer and probe set evaluated in this study has been designed to specifically detect $A(H 1) v$ influenza in a one-step RT-PCR assay.

\section{HPA (N1)v assay}

The influenza $A(N 1) v$ real-time assay (HPA) is a two-step TaqMan PCR assay incorporating oligonucleotide primers and a dual-labelled MGB TaqMan probe for the detection of the NA gene of influenza $A(H 1 N 1) v$ viruses and the positive control virus A/Aragon/3218/2008 [5]. The assay has been designed to be performed in conjunction with the influenza $A(H 1) v$ specific assay, to provide confirmation of diagnosis of influenza $A(H 1 N 1) v$ virus infection.

\section{S-OIV assay}

The swine-origin influenza virus (S-OIV) assay (National Virus Reference Laboratory, NVRL, Dublin) is a real-time one-step RTPCR assay containing primers and a dual-labelled hydrolysis probe targeting the $M$ gene of influenza $A$ viruses other than seasonal $A(H 1 N 1)$ and $A(H 3 N 2)$ viruses [6].

\section{Methods}

Respiratory samples (85 nose or throat swabs) were submitted as part of the influenza $A(H 1 N 1) v$ virus investigation in the United Kingdom. Of these, 43 influenza A-positive, untypable, $M$ gene sequence-confirmed cases of influenza $A(H 1 N 1) v$, and $42 A(H 1 N 1)$ $v$-negative samples containing seasonal influenza $A(H 1 N 1)$, $A(H 3 N 2)$ or influenza $B$, or negative for influenza viruses, were analysed using the real-time assays. In addition, specificity was evaluated using representative influenza $A$ viruses of HA subtype $\mathrm{H} 5, \mathrm{H} 6, \mathrm{H} 7$ and $\mathrm{H9}$, and a panel of non-related respiratory viruses: respiratory syncytial viruses (RSV A and RSV B), parainfluenza viruses, rhinoviruses, human metapneumoviruses (hMPV) and corona viruses. Viral RNA was purified from clinical samples and viral cultures using the Biomerieux NucliSens easyMAG system.

Specimens were tested according to the protocol provided for each assay. All assays were run on an ABI Taqman 7500 Fast Thermal Cycler in standard (one-step assays) or Fast (two-step) mode. All samples were tested in duplicate. Discrepant results were confirmed by repeat testing. Ct values of $<40.00$ were considered to be positive for detection of viral RNA.

\section{Results}

The relative sensitivity of the assays was compared by analysing a 10-fold dilution series of A/England/195/2009(H1N1)v (nose swab sample). 
No cross-reaction was observed when the four real-time assays were used to test 22 seasonal influenza viruses, or other respiratory viruses. A panel of representative influenza A viruses of different subtypes was also analysed (Table 2).

The HPA (H1)v and CDC (H1)v specific assays showed no crossreactivity with any of the other influenza $A$ subtypes analysed. The HPA (N1)v confirmatory assay detected one influenza $A(H 5 N 1)$ virus, but showed no cross-reactivity with other subtype viruses. The S-OIV assay showed cross-reactivity with all of the influenza A viruses analysed.

When 43 true positive samples were analysed, 36 were positive in all four real-time PCR assays (Table 3 ).

Four false negative and two equivocal results were observed with the $\mathrm{CDC}(\mathrm{H} 1) v$ assay. One equivocal result was observed with the S-OIV assay. Two samples were negative with either the HPA $(\mathrm{H} 1) \mathrm{v}$ or (N1)v assays, but when these assays were performed in parallel, as recommended, one false negative result was observed. No false-positives were detected in the 42 influenza $A(H 1 N 1) v$ virus-negative samples with any of the four real-time assays.

The $\mathrm{Ct}$ values obtained by analyses with the real-time assays of the 43 confirmed influenza A (H1N1)v virus samples are shown in Figures 1a-c. A total of 42 true negative and 43 true positive samples were tested in all assays. Comparison of the HPA $(\mathrm{H} 1) v$ and $\mathrm{CDC}(\mathrm{H} 1) \mathrm{v}$ assays showed that of the 43 true positives tested, 41 were detected in the HPA ( 11$)$ v assay (Figure $1 \mathrm{la})$. Thirty seven were positive and 2 equivocal in the CDC (H1)v assay. Three samples positive in HPA ( $\mathrm{H} 1)$ v assay were negative in the $\mathrm{CDC}$ assay and 1 sample positive in the HPA (H1)v assay was equivocal in the CDC (H1)v assay.

Of the 43 true positives, 41 were positive in the HPA $(\mathrm{H} 1) \mathrm{v}$ assay and 42 in the S-OIV assay (Figure $1 \mathrm{~b}$ ). One sample gave an equivocal result with the S-OIV assay.

Comparison of the HPA ( $\mathrm{H} 1)$ v diagnostic assay with the HPA (N1)v confirmatory assay demonstrated that the two assays correlate well, with a correlation coefficient of $r=0.97$ (Figure $1 \mathrm{c}$ ).

The precision of the HPA (H1)v and (N1)v real-time assays was assessed by the coefficient of variation (CV) and standard deviation (SD) of the replicate Ct measurements ( $n=37$ and $n=9$ respectively) for the assay-positive control on diagnostic assay runs. The CV for the mean Ct values obtained with the (H1)v and (N1)v assaypositive controls was $3 \%$ and $2 \%$ respectively.

T A B L E 1

End-point detection of A/England/195/2009 (H1N1)v by four real-time PCR assays

\begin{tabular}{|c|c|c|c|c|}
\hline \multirow[b]{2}{*}{ Dilution A/Eng/195/2009(H1N1)v } & \multicolumn{4}{|c|}{ Mean Ct values } \\
\hline & HPA (H1)v & $\operatorname{CDC}(\mathrm{H} 1) \mathrm{v}$ & $\begin{array}{l}\text { HPA } \\
\text { (N1)v }\end{array}$ & S- OIV \\
\hline $1.00 \mathrm{E}-03$ & 18.35 & 24.85 & 23.50 & 21.85 \\
\hline $1.00 \mathrm{E}-04$ & 21.35 & 28.25 & 27.15 & 25.30 \\
\hline $1.00 \mathrm{E}-05$ & 24.60 & 31.75 & 30.60 & 28.45 \\
\hline $1.00 \mathrm{E}-06$ & 27.95 & 35.20 & 34.25 & 31.70 \\
\hline $1.00 \mathrm{E}-07$ & 30.65 & 38.70 & 36.10 & 37.60 \\
\hline $1.00 \mathrm{E}-08$ & 32.95 & Neg & 38.80 & 36.85 \\
\hline $1.00 \mathrm{E}-09$ & Neg & Neg & Neg & Neg \\
\hline
\end{tabular}

T A B L E 2

Specificity of four real-time PCR assays with representative influenza A subtype virus isolates

\begin{tabular}{|l|c|c|c|}
\hline Influenza A virus subtype & HPA (H1)v & CDC (H1)v & HPA (N1)v \\
\hline A/Cambodia/R0405050/2007 RG H5N1 & Neg & Neg & Neg \\
\hline A/Indonesia/6/2005 H5N1 & Neg & Neg & Neg.77 \\
\hline A/Chicken/Turkiye/Av05/2006 H5N1 & Neg & Neg & Neg \\
\hline A/Vietnam/1203/2004 H5N1 & Neg & Neg & Neg \\
\hline A/Duck/Singapore-Q/F119-3/97 H5N3 & Neg & Neg & Neg \\
\hline A Turkey/England/198/2009 H6N1 & Neg & Neg & Neg \\
\hline A/AfricanStarling/Q-England/983/79 H7N1 & Neg & Neg & Neg \\
\hline A/Chicken/Wales/306/2007 H7N2 & Neg & Neg & not done \\
\hline A/Quail/HongKong/G1/97 H9N2 & & 30.53 \\
\hline
\end{tabular}

a derived by reverse genetics 
T A B L E 3

Comparison of HPA (H1)v, CDC (H1)v, HPA (N1)v, and S-OIV real-time PCR assays on sequence confirmed swine-lineage samples

\begin{tabular}{|l|c|c|c|c|}
\hline Number of samples & HPA (H1)v & CDC (H1)v & HPA (N1)v & S-0IV \\
\hline 36 & + & + & + & + \\
\hline 1 & + & - & + & Equiv $^{\star}$ \\
\hline 2 & + & - & + & + \\
\hline 1 & - & + & + & + \\
\hline 1 & - & - & - & + \\
\hline 1 & + & Equiv $^{*}$ & + & + \\
\hline 1 & + & Equiv $^{*}$ & - & + \\
\hline Sensitivity (\%) & 95.4 & 90.7 & 95.4 & 100 \\
\hline
\end{tabular}

* Weak positive in one replicate

F I G U R E 1 A

Comparison of HPA (H1)v and CDC (H1)v assay Ct values

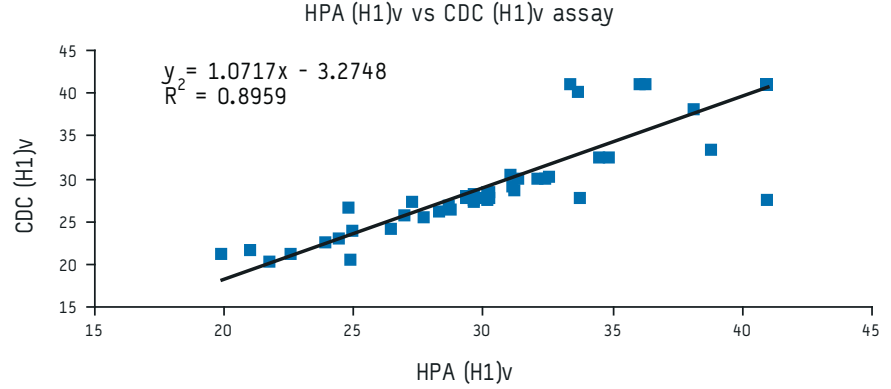

F I G U R E 1 B

Comparison HPA (H1)v and S-OIV assay Ct values

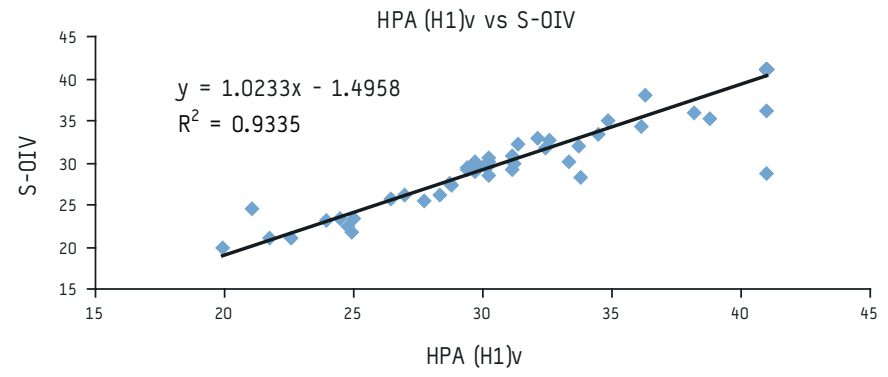

F I G U R E 1 C

Correlation of Ct values obtained with HPA (H1)v and (N1) v assays

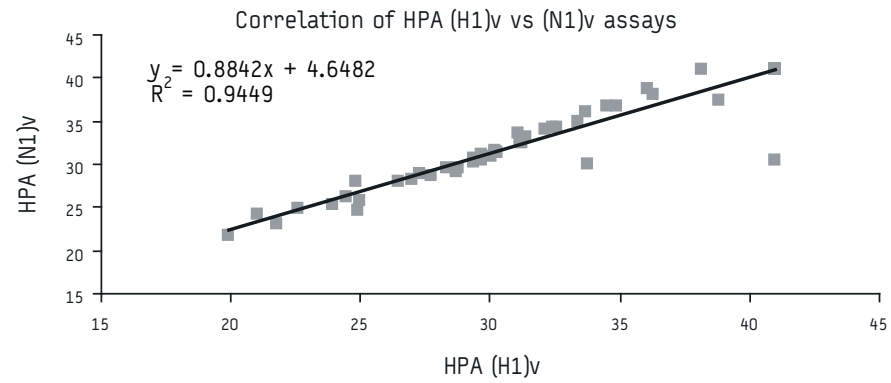

\section{Conclusions}

The sensitivities and specificities of the four assays were in a similar range and suitable for diagnostic use. The HPA (H1)v and the S-OIV assays were the most sensitive assays for use as a first line test, but the S-OIV assay was less specific, detecting all avian subtypes of influenza A viruses tested. For confirmation, an assay in another gene such as the HPA (N1)v could be employed. The results obtained with the HPA ( $\mathrm{H} 1)$ v and (N1)v assays correlated well and, in addition, intra-assay variability of the HPA (H1)v and (N1)v assays was shown to be acceptable with values for the coefficient of variation $(\mathrm{CV})<5 \%$.

Because the security of a diagnostic result for influenza $A(\mathrm{H} 1 \mathrm{~N} 1)$ $\checkmark$ virus is important for public health actions, the use of primary detection and confirmatory assays as described here is appropriate. The use of the HPA ( $\mathrm{H} 1) v$ and $(\mathrm{N} 1) v$ assays together provides rapid and accurate assessment of confirmed cases, and enables appropriate management of patients.

\section{Acknowledgements}

The authors would like to acknowledge the contribution of the member laboratories of the Regional Microbiology Network, HPA, and participating Royal College of General Practitioners practices, in submitting the clinical samples evaluated in this study. The S-OIV real-time PCR assay protocol and primer and probe sequences were kindly provided by M Carr and S Coughlan (NVRL, Dublin). The influenza $A(H 1 N 1)$ virus A/Aragon/3218/2008 was kindly provided by the Director of the National Centre for Microbiology, Instituto de Salud Carlos III, Madrid, Spain. Technical assistance in performing the evaluation was provided by $T$ Talts and $C$ Amar.

\section{References}

1. Novel Swine-Origin Influenza A (H1N1) Virus Investigation Team. Emergence of a Novel Swine-Origin Influenza A (H1N1) Virus in Humans. N Engl J Med. 22 May 2009. [Epub ahead of print].

2. Centers for Disease Control and Prevention (CDC). Swine influenza A (H1N1) infection in two children - Southern California, March-April 2009. MMWR Morb Mortal Wkly Rep 2009;58(15):400-2

3. Health Protection Agency (HPA). Swine-Lineage Influenza A H1 Specific Fast Real Time PCR. National Standard Methods - Virology. VSOP 29, Issue 2, May 2009. Available from: http://www.hpa-standardmethods.org.uk/pdf sops.asp

4. World Health Organization (WHO). CDC protocol of realtime RTPCR for influenza A (H1N1). 30 April 2009. Available from: http://www.who.int/csr/resources/ publications/swineflu/realtimeptpcr/en/index.html

5. Health Protection Agency (HPA). Swine-lineage Influenza A N1 Fast Real Time Confirmatory PCR assay. National Standard Methods - Virology. VSOP 48, Issue 1, May 2009. Available from: http://www.hpa-standardmethods.org.uk/pdf_sops. asp

6. Personal communication from Dr Michael Carr, National Virus Reference Laboratory (NVRL), Dublin, 22 May 2009.

This article was published on 4 June 2009.

Citation style for this article: Ellis J, Iturriza M, Allen R, Bermingham A, Brown K, Gray J, Brown D. Evaluation of four real-time PCR assays for detection of influenza A(H1N1)v viruses. Euro Surveill. 2009;14(22):pii=19230. Available online: http://www. eurosurveillance.org/ViewArticle.aspx?ArticleId $=19230$

*Authors' correction:

The name of the third author was corrected on 13 June 2012 at the request of the authors. 